\title{
Bladder Sphincter Dysfunction
}

National Cancer Institute

\section{Source}

National Cancer Institute. Bladder Sphincter Dysfunction. NCI Thesaurus. Code C123208.

Non-coordinated, reflexive contraction of the bladder and sphincter relaxation. 